\title{
AULA INVERTIDA EN TIEMPOS EMERGENTES COVID-19
}

\section{FLIPPED CLASSROOM IN COVID-19 EMERGING TIMES}

\author{
Eulalia Beatriz Becerra García ${ }^{1}$ \\ Karina Belén Quintana Pacheco² \\ Edisson Iván Reyes Pacheco ${ }^{3}$
}

Recibido: 2020-01-02 / Revisado: 2020-04-15 / Aceptado: 2020-05-05 / Publicado: 2020-07-01

Forma sugerida de citar: Becerra-García, E. B., Quintana-Pacheco, K. B. y Reyes-Pacheco, E. I. (2020). Aula invertida en tiempos emergentes Covid-19. Retos de la Ciencia. 4(9), pp. 24-36. https://doi.org/10.53877/rc.4.9.20200701.03

\section{RESUMEN}

La comunicación horizontal en la virtualidad requiere de procesos sincrónicos y asincrónicos que beneficien a los estudiantes y docentes al cumplir con las actividades inherentes a su rol. Pero, en circunstancias atípicas es necesario proponer el aula invertida como una estrategia de acompañamiento para el avance de la construcción del trabajo de monografía de los estudiantes del Bachillerato Internacional en Español Categoría 1. A través de la sistematización de la experiencia profesional. La guía de acompañamiento docente tiene como finalidad sistematizar el avance de la construcción del trabajo docente a través del aula invertida en función de un cronograma de actividades y las herramientas necesarias para la exploración como estrategia didáctica para mejorar el proceso de enseñanza-aprendizaje. Se aplicó una ficha de sistematización de aprendizajes, que evidenció que los estudiantes tienen dificultades para realizar sus actividades sincrónicas, por lo que, se propone el uso del aula invertida como alternativa de solución al problema abordado.

Palabras clave: aula invertida, comunicación, enseñanza-aprendizaje, habilidades.

\section{ABSTRACT}

Horizontal communication in virtuality requires synchronous and asynchronous processes that benefit students and teachers by fulfilling the activities inherent to

\footnotetext{
${ }^{1}$ Magister en Docencia y Currículo para la Educación Superior. Docente de la Facultad de Educación y Desarrollo Social de la Universidad Tecnológica Indoamérica. Ambato-Ecuador. E-mail: eulaliabecerra@uti.edu.ec / https://orcid.org/0000-0002-0677-7393

${ }^{2}$ Licenciada en Comunicación Social Mención Periodismo de Investigación. Maestrante en Innovación en Educación. Pontificia Universidad Católica del Ecuador sede Ambato-Ecuador. E-mail: karina.b.quintana.p@pucesa.edu.ec / https://orcid.org/0000-0001-5095-5305

3 Ingeniero en Electrónica y Comunicaciones. Maestrante en Pedagogía con mención en Educación Técnica y Tecnológica. Pontificia Universidad Católica del Ecuador sede Ambato-Ecuador. E-mail: edisson.i.reyes.p@pucesa.edu.ec / https://orcid.org/0000-0002-8970-9977
} 
their role. But, in atypical circumstances, it is necessary to propose the flipped classroom as an accompaniment strategy for the advancement of the construction of the monograph work of the students of the International Baccalaureate in Spanish Category 1. Through the systematization of professional experience. The purpose of the teaching accompaniment guide is to systematize the progress of the construction of the teaching work through the flipped classroom based on a schedule of activities and the tools necessary for exploration as a didactic strategy to improve the teaching-learning process. A learning systematization sheet was applied, which showed that students have difficulties to carry out their synchronous activities, therefore, the use of the flipped classroom is proposed as an alternative solution to the problem addressed.

Keywords: flipped classroom, communication, teaching-learning, skills.

\section{INTRODUCCIÓN}

El desarrollo de un trabajo original, independiente, de carácter analítico y argumentativo ha sido uno de los esquemas utilizados por el sistema educativo "[...] los estudiantes de tercer año de bachillerato ya tienen su cronograma para la elaboración de una monografía o proyecto de grado, que corresponderá al $10 \%$ de la nota final" (Guía ser Bachiller: País: La Hora Noticias de Ecuador, Sus Provincias y El Mundo), con esta producción se pretende promover la investigación de un tema de interés en diversas disciplinas académicas.

El Consejo de Educación superior, (2010), dispuso que el ingreso a las instituciones de educación superior debía estar regulado por el Sistema de Nivelación y Admisión (SNNA), es entonces que desde el 2011 estableció el Examen Nacional de Educación Superior (ENES). (Ser Bachiller: Desde Su Inicio La Prueba Ha Tenido Cinco Cambios)

El programa del Bachillerato Internacional en su página web promueve la adhesión de los estudiantes al perfil de la comunidad $\mathrm{BI}$ con atributos como: "pensadores, buenos comunicadores, de pensamiento crítico, reflexivos, indagadores, audaces, entre otros" (International Baccalaureate Organization, 2013); de esta manera la producción de la monografía como trabajo independiente permitirá desarrollar capacidades críticas, argumentativas e investigativas que evidencien habilidades cognitivas y meta-cognitivas.

Los ambientes educativos mejoran la comunicación horizontal a través de la información que se presenta en Internet. De esta manera se asegura que quienes exploran el mundo virtual acceden de forma directa al conocimiento. Castri (2010), afirma: "La tecnología remite hoy no a unos aparatos sino a un ecosistema comunicativo" (p. 46).

La tecnología ha beneficiado al proceso educativo, social, y cultural. "La Web es ahora un espacio necesario, en el cual el usuario ya no sólo utiliza información, sino que también, produce contenidos con recursos" (Maria y Ovide, 2011, p. 37). En la tecnología existen dos tipos de personas, según Fernández-Plaza, et. al., (2016): el nativo e inmigrante digital. El primero domina y experimenta el mundo tecnológico por lo que su participación debe enfocarse paralelamente a la concepción del mundo real; en cambio el segundo tiene dificultades para explorar y familiarizarse con este nuevo sistema de información, en ellos hay que fomentar la participación protagonista para promover su cultura participativa.

En consecuencia, la aplicación de tecnología en la educación no solo instaura un nuevo modelo educativo moderno, sino que promueve una participación 
constante de quienes intervienen en estos procesos para desarrollar habilidades digitales, académicas que configuren en el individuo la capacidad de aprender y convivir en un entorno en el que su interacción será continua. "La computadora es el elemento mediador por excelencia de nuestro tiempo histórico" (Ibarra y Llata, 2010, p. 5).

En la educación el uso de la tecnología es constante, el uso de dispositivos, herramientas tecnológicas figuran como una estrategia, por ende su adquisición y uso está regulada por las necesidades de cada individuo "el impacto de las TIC, dentro de la sociedad del conocimiento ha traído grandes cambios, respecto a forma y contenido" (Hernández, 2017, p. 329). En el ámbito educativo existe la necesidad de modernizar las aulas, acorde a las necesidades del contexto.

La tecnología tiene un impacto en la reforma educativa, según Granja (2015), requiere que el aula, se transforme para que el protagonista sea el alumno y el docente se convierta en su orientador, por esta razón la construcción del conocimiento en cada ser humano, se adquirirá de acuerdo a la percepción de su realidad; de esta manera, la organiza y le da sentido, bajo este criterio el gestor del conocimiento debe ser el propio estudiante pues la interpretación le permitirá encontrar una explicación de lo que conoce y así edificará las bases de su aprendizaje. "El aula como comunidad de aprendizaje representa una nueva manera de entender este proceso generando cambios en las formas de organización de la enseñanza" (Rojas et. al., 2014, p. 233). Téngase en cuenta que, el ser humano se desarrolla en la práctica formativa (Castillo, 2015. 9)

En este sentido, la inclusión de la tecnología en la educación virtual, como consecuencia de la pandemia del COVID 19, generó en los docentes y estudiantes un nuevo modelo de enseñanza-aprendizaje; el mismo que fortaleció el trabajo en plataformas virtuales, y el uso de herramientas tecnológicas como pilar de desarrollo comunicacional entre alumnos y maestros. La enseñanza es parte del quehacer educativo y se adapta a las necesidades y circunstancias cambiantes del ambiente "el deseo por ampliar la cobertura y por mejorar la calidad haciendo de ella un auténtico espacio de formación" (Unigarro, 2004, p. 5).

\section{METODOLOGÍA}

La suspensión de clases virtuales como consecuencia del confinamiento generado por la pandemia del COVID 19, paralizó los procesos educativos hasta presentar un plan alterno por la emergencia sanitaria; sin embargo, los estudiantes que cursaban tercero de bachillerato en el programa del diploma del Bachillerato Internacional, no podían detener sus actividades, pues las mismas debían ser entregadas en el mes de marzo para la evaluación externa, la monografía es una producción creativa y crítica del estudiante que se califica de forma externa; por lo que su desarrollo debía continuar y para ello se planificó mediante el modelo del aula invertida un plan de acompañamiento con actividades sincrónicas y asincrónicas para que dicha actividad no pierda continuidad.

Usando una ficha de sistematización de aprendizaje, se evidenció que los estudiantes no podían proceder con su trabajo solo con actividades asincrónicas; por lo que la propuesta del aula invertida constituyó una alternativa viable, para que los dos estudiantes de treinta y ocho que cursaban el programa $\mathrm{BI}$, que eligieron la asignatura de español A: Literatura NS como disciplina para realizar su trabajo investigativo en la convocatoria mayo 2020, puedan concluir su proceso de la mejor manera. Debido a las circunstancias tanto estudiantes como docentes se acoplaron 
a este nuevo modelo de enseñanza-aprendizaje para que su monografía se entregue en el tiempo previsto y su calidad se ajuste a los criterios de evaluación en los estándares más altos.

Usando la tecnología se definieron etapas para la producción y culminación del proceso de monografía, en primera instancia se compartió una serie de recursos bibliográficos para que la exploración tenga antecedentes del tema y se pueda justificar la importancia del trabajo, esto se lo realizó en la etapa asincrónica a través de aula virtual en la plataforma del aula invertida, de forma seguida se realizaron sesiones de reflexión en la plataforma meet para intercambiar ideas y argumentos que justifiquen el trabajo en cada una de sus partes: introducción, desarrollo y conclusiones.

Usando espacios y herramientas virtuales se presentó el plan de acompañamiento de la monografía dividido en actividades sincrónicas y asincrónicas mediadas por sesiones de consulta y reflexión.

\section{a) Sesiones de reflexión formal}

Son las sesiones obligatorias que deben registrarse en el formulario de reflexión sobre la planificación y el progreso, con relación al documento Organización del Bachillerato Internacional del 2017. La duración recomendada es de entre 20 y 30 minutos. Las sesiones obligatorias se enfocan en la revisión de progresos realizados y en el planteo de objetivos concisos para que el alumno continúe con el desarrollo de su monografía y se desarrolla mediante diálogos por preguntas que el supervisor considere importantes. En este primer encuentro el rol del supervisor es encaminar a que el estudiante planteé su tema y pregunta y defina su método de investigación.

\section{b) Sesiones de consulta}

Esta sesión está diseñada para que el supervisor de monografía atienda las necesidades particulares de cada alumno, es decir brinda "una educación que responda con solvencia a la diversidad en su más amplia significación y sentido" (Yépez y Castillo, 2020, p. 14), y desarrolle un proceso de supervisión solvente, y efectivo como considera la Organización del Bachillerato Internacional (2017); el tiempo de supervisión puede consistir simplemente en una reunión de consulta ocasional de 10 minutos para discutir un cronograma o aclarar un comentario del supervisor.

Estos entornos de consulta se benefician al estudiante para aclarar dudas puntuales sobre su proceso, se pueden registrar cambios según su progreso y también representan un espacio de discusión para inferir, interpretar y contextualizar los aspectos formales del trabajo de acuerdo con los criterios de la asignatura.

Considerando los enfoques y el tratamiento que el programa del diploma sugiere para la guía y la ejecución de la monografía, existe una gran cantidad de métodos de enseñanza aprendizaje aplicables para la construcción del conocimiento de esta índole. Después de analizar la guía actualizada de monografía 2020 diseñada por la Organización del Bachillerato Internacional, se determinó que el aula invertida es un método que se ajusta a las necesidades que el programa requiere, debido a que la evaluación del trabajo final lo realiza un ente externo, pasando de esta manera el supervisor a ser estrictamente un guía, tutor, mentor de los trabajos de monografía, por lo que la planificación de guía de monografía 2016 vigente se ajusta a la misma. "En la aplicación del modelo pedagógico de clase invertida, es necesario que el profesor cambie su rol de actor, por el de orientador" (Albornoz,et al, 2020, p.6).

\section{Aula invertida}

Hernández y Flores (2017), refieren que los modelos educativos tradicionales se caracterizan por estar ajustados al rol protagónico del docente y los contenidos 
dentro del aula (Hernández-Silva y Flores, 2017, p. 194). No obstante, en el modelo del aula invertida esta cosmovisión cambia, para que el proceso educativo transforme su esquema e invierta el rol participativo de cada miembro en el accionar de la enseñanza-aprendizaje. "Al ser el docente uno de los principales actores del proceso educativo, asume directamente la responsabilidad y el compromiso de gestar la transformación social, a través de la transformación individual y colectiva de los estudiantes" (Castillo, 2013, pp. 2-3)

Con el aula invertida el estudiante es el protagonista en la construcción de su proyecto, pero la eficacia de este se ve reflejada en la sistemática guía por parte del docente supervisor. Akçayır (2018), señala:

Las ventajas en términos de los resultados de aprendizaje de los estudiantes (como satisfacción, compromiso y motivación) se agrupan en esta categoría. Más de la mitad de los estudios (52\%) informaron que el uso del modelo invertido mejora el rendimiento de aprendizaje de los estudiantes, cuando se mide mediante GPA, puntajes de exámenes estandarizados y calificaciones de cursos. Según estos resultados, una de las ventajas más significativas de este modelo es que ayuda a mejorar el rendimiento del aprendizaje, que es uno de los elementos clave de la educación de calidad. (p. 338)

En tiempos de Covid 19 y en función del documento expreso por el (BI), es necesario adoptar dos tipos de estrategias virtuales que se enfoquen didácticamente en el aprendizaje colaborativo., en la cual se espera que ocurran formas particulares de interacción, que producirán mecanismos de aprendizaje, que posiblemente conduzcan al logro de un aprendizaje" (Collazos, 2006, p. 63).

\section{Estrategia virtual sincrónica}

Constituye una estrategia que articula la relación entre supervisor- alumno, por lo que se utilizará como el modo de relación para las sesiones de reflexión forma, Casal (2017), señala que:

Es evidente que el uso de herramientas de comunicación en tiempo real refuerza la interacción profesional, las relaciones socioemocionales y las interacciones personales; en definitiva, son una herramienta que facilita y refuerza la comunicación interpersonal entre los usuarios (p. 448).

\section{Estrategia virtual asincrónica}

Esta estrategia organiza un medio de comunicación con los alumnos y hace las veces de mediador en la construcción del conocimiento, en este caso se considera una estrategia eficaz y adyacente a los lineamientos de la guía (BI), por esta razón aprovecharemos sus funcionalidades como estrategia para acompañar a los estudiantes en la construcción de su monografía. Hope y Ca (2016), afirma que: "la comunicación asincrónica se establece entre dos o más personas de manera diferida en el tiempo, es decir, cuando no existe coincidencia temporal" (p. 20).

A continuación, se presenta en forma secuencial la propuesta de un plan de acompañamiento docente para el avance de la construcción del trabajo de monografía de los estudiantes del Bachillerato Internacional (BI) en Español categoría 1, en la pandemia del COVID-19, por medio de una planificación inversa con estrategia virtual didáctica; enmarcada a la guía actualizada de monografía 2020 diseñada por la Organización del Bachillerato Internacional: con la que se pretende sistematizar las tutorías de acompañamiento docente para la construcción de monografías del programa del diploma del bachillerato internacional.

Planificación inversa de guía docente para el proceso de supervisión de la monografía de español categoría 1 del (BI) en tiempos emergentes (COVID-19)

Objetivo: Guiar de forma sistemática el desarrollo de la monografía de español categoría 1 mediante estrategias sincrónicas y asincrónicas de enseñanza para que 
los estudiantes puedan alcanzar calificación "A" en este componente de evaluación del programa del diploma del Bachillerato Internacional.

Perfil de la comunidad de aprendizaje del (BI): El objetivo fundamental de los programas del Bachillerato Internacional $(\mathrm{BI})$ es formar personas con mentalidad internacional que, conscientes de la condición que las une como seres humanos y de la responsabilidad que comparten de velar por el planeta, contribuyan a crear un mundo mejor y más pacífico, esforzados por ser: indagadores, informados e instruidos, pensadores, buenos comunicadores, íntegros, de mentalidad abierta, solidarios, audaces, equilibrados, reflexivos.

Actividades previas a las sesiones de reflexión formal (Enseñanza virtual, asincrónica)

\section{a) Actividades del supervisor}

Primera Sesión. - Animar a los alumnos a incluir en sus espacios de reflexión ejemplos de exploración inicial de temas, posibles fuentes y métodos, preguntas de investigación preliminares y sus reacciones personales a las cuestiones.

Segunda Sesión. - Proponer un esquema de investigación resaltando algunos aspectos literarios y lingüísticos para desarrollar.

Tercera Sesión. - Revisar el trabajo de investigación, verificar que se dé cumplimiento a lo planificado y se cumplan con los criterios de evaluación propuestos en la guía de monografía.

\section{b) Actividades del alumno}

En la Tabla 1, se expone en forma secuencial las actividades que el alumno debe considerar con anterioridad al desarrollo de las sesiones de reflexión formal, apoyando su trabajo con los recursos virtuales didácticos. El estudiante completará estas actividades en 25 días.

\section{Tabla 1:}

Actividades del alumno previas a las sesiones de reflexión formal

\begin{tabular}{ll}
\hline Actividades del alumno & Recursos didácticos \\
\hline Primera sesión. & Autores prescritos $(\mathrm{BI}):$
\end{tabular}

1. Revisa la lista de autores prescritos de (BI).

2. Elije al menos tres obras literarias para examinar cada una de ellas, de esta manera se valorar su pertinencia.

3. Selecciona los enfoques y plantea posibles preguntas de investigación.
Adoum. J; Alberti, R; Aleixandre, V; Altolaguirre, M y Arcipreste De Hita, J. (2011)

Enfoques (BI):

De pensamiento: Habilidades críticas, creativas y de transferencia.

De autogestión: Habilidades organizativas, afectivas y de reflexión.

De investigación: Habilidades de gestión de la información y de alfabetización mediática.

Sociales: Habilidades de colaboración y comunicación.

Guía de monografía:

Organización del Bachillerato Internacional (2017).

Monografías de convocatorias pasadas:

Título de la monografía.

Informe y declaración del supervisor. 
Formulario de evaluación.

Segunda sesión

1. Concreta pregunta investigación centrada y apropiada.

2. Profundiza de manera significativa

su investigación, registra pruebas, información o datos adecuados en el espacio de reflexión.

3. Revisa y consolida las metodologías que está utilizando.

4. Formula argumentos basados en las pruebas que hayan obtenido y fundamenta las ideas que plantea justificando con bibliografía para la investigación.

Tercera sesión

1. Presenta su trabajo de investigación, domina el contenido desarrollado y lo defiende con argumentos sólidos y bien fundamentados con bibliografía pertinente para la tarea.
Plantilla de esquema literario:

Elementos lingüísticos y literarios:

una 1. Análisis de personajes: por su acción, profundidad

psicológica, por su naturaleza. (Identidad, características físicas, psicológicas, relaciones sociales).

2. Análisis de espacios: físicos, psicológicos, sociales.

3. Ambiente y atmósfera de la obra: tensión, misterio, hostil, tranquilidad, etc.

4. Tipo de narrador: omnisciente, testigo, protagonista.

5. Perspectivas narrativa: heterodiegética, auto-diegética, homocigótica.

6. Focalización: interna, externa, cero.

7. Tiempo narrativo: de la historia (cronología-anacrónica); histórico (contextual); del relato (ab-ovo, in media res, in extrema res).

8. Funciones del lenguaje: emotiva, poética, referencial, apelativa, metalingüística, fática.

9. Tonos: irónico, sarcástico, etc.

10. Tipo de lenguaje: formal, coloquial, culto, vulgar, etc.

11. Figuras literarias: fonológicas, léxico semánticas.

12. Símbolos: tangibles e intangibles.

13. Análisis de fondo y forma (estructura interna y externa).

Consejos para plantear preguntas: literales, inferenciales y críticas:

Fuentes y textos con crítica literaria sobre la obra que se estudiará, otras obras y autores que hablen del tema:

Coherencia y cohesión textual:

Normas APA sexta edición:

Carga la versión final de monografía en la plataforma classroom para su revisión final:

Revisión de trabajo monográfico en estructura y forma:

Fuente: Elaboración propia.

\section{Sesiones alumno-supervisor (Enseñanza virtual sincrónica)}

\section{a) Sesiones de consulta}

Primera Sesión. - Conversatorio en Google Meet sobre las obras leídas, la propuesta de investigación, los métodos que se han identificado para la exploración y las dificultades encontradas en el proceso.

Discusión de la propuesta de investigación literaria con ideas concretas de: ¿lo que se hará?, ¿para qué se hará?, ¿cómo se hará? (Análisis de personajes, narradores, espacios, elementos estilísticos y lingüísticos). Esquematización de la información que se propone trabajar. Se crea un documento compartido en google 
drive y se recomienda a que el alumno envié a su supervisor un esquema literario de su propuesta de investigación, el estudiante debe dejar en claro lo que analizará.

Segunda Sesión. - Conversatorio en Google Meet. Análisis de la información y fuentes bibliográficas que se utilizarán, revisión de estructura sintáctica y semántica generada para la investigación. Estructura de la monografía: su forma y fondo; número de capítulos, temas sub-temas, ¿cómo se aplicará la exploración y habilidades de comprensión e inferencias de la obra en estudio?

Plantear una pregunta de investigación abierta, con componentes literarios. Analizar elementos literarios contextuales, conceptuales presentes en la obra seleccionada y en otras referencias literarias. Redactar dos párrafos con coherencia y cohesión demostrando su conocimiento y comprensión de la obra a estudiar con el apoyo de otros autores y obras. Proponer un índice de desarrollo de la investigación para esquematizar la monografía.

Tercera Sesión. - Conversatorio en Google Meet para exponer y defender su trabajo monográfico de forma oral y recibir una retroalimentación según los criterios de evaluación de la monografía y las implicaciones lingüísticas y literarias de la asignatura.

Defiende la introducción de la monografía en 500 palabras, presentando: antecedentes literarios, método, justificación, importancia, pregunta, tema de investigación. Justifica el cuerpo de la monografía planteándose algunos cuestionamientos literarios para generar conclusiones parciales en cada sección que respondan a la pregunta y tema de investigación. Corrige el trabajo monográfico según las observaciones planteadas por el supervisor. Revisa coherencia, cohesión, gramática y ortografía. Redacta las conclusiones de la investigación atendiendo al tema y pregunta de investigación.

\section{b) Sesiones de reflexión formal}

La Tabla 2 muestra las actividades que el alumno-supervisor aborda en el desarrollo de las sesiones formales $(1,2,3)$. Para estas actividades se ocuparán 90 minutos.

Tabla 2:

Actividades alumno-supervisor en sesiones formales

Sesiones de reflexión formal Estrategia virtual de enseñanza

Primera sesión de reflexión.

1. Repaso de los requisitos y los criterios de evaluación para la asignatura.

2. Repaso de las implicaciones éticas y legales, para el proceso de monografía y entrega.

3. Diálogo sobre los enfoques posibles y los problemas que podrían surgir.

4. Discusión sobre las estrategias para desarrollar las ideas del alumno para la monografía y ampliar la investigación para que el trabajo empiece a tomar forma.

5. Preguntas estimulantes y de sondeo
Conversatorio en Google Meet:

1. Definición de antecedentes, justificación, importancia, tema, pregunta de investigación y método.

2. Preguntas sobre los elementos que se incluirán en la monografía para posteriormente esquematizarlos.

3. Envío del pdf de reflexiones por correo electrónico. 
que ayuden a los alumnos a aclarar sus ideas; esto debe llevar al desarrollo de la pregunta de investigación provisoria del alumno.

6. Resumen de los pasos siguientes que el alumno deberá dar para refinar su pregunta; dar forma a la investigación y un cronograma escrito.

Sesión de reflexión intermedia

1. Revisión del avance de la monografía y retroalimentación de los aspectos positivos y negativos.

2. Preguntas de indagación para que el estudiante demuestre su comprensión y análisis de la obra.

Sesión de reflexión final

1. Los alumnos pueden demostrar lo que han aprendido sobre el tema, el proceso de investigación que han seguido, su propio aprendizaje, y también resumir nuevas preguntas que han descubierto.
Conversatorio en Google Meet:

1. Definición de aspectos metodológicos y revisión de elementos literarios y lingüísticos que se considerarán para la investigación.

2. Revisión de lenguaje y organización de ideas.

3. Envío del pdf de reflexiones por correo electrónico.

Conversatorio en Google Meet:

1. Envío del pdf de reflexiones por correo electrónico.

Fuente: Elaboración propia.

Actividades subsiguientes a las sesiones de reflexión formal (Enseñanza virtual asincrónica)

\section{a) Actividades del alumno}

en la Tabla 3 se da a conocer las actividades que el alumno debe realizar al finalizar cada sesión de reflexión formal. Se utilizarán 21 días para estas actividades.

Tabla 3:

Actividades del alumno subsiguientes a las sesiones de reflexión formal

Actividades del alumno

Primera sesión.

1. Indagar otros autores que hablen del tema que se pretende estudiar, que sean contemporáneos, que pertenezcan al mismo movimiento literario.

2. Revisión de bibliografía, artículos científicos, crítica literaria.
Recursos educativos

Gestores de búsqueda:

Scholar, scielo, scopus.

Formulario:

Monografía: Formulario de reflexión sobre la planificación y el progreso (2018).

3. El estudiante explica las razones para la 
selección de la obra literaria a estudiar. qué preguntas preliminares surgieron de su lectura, qué quiere investigar y cómo, qué método de investigación le ayudará a cumplirlo.

4. Avanzar en el desarrollo de las actividades previas a la sesión de reflexión intermedia.

5. El estudiante indica el progreso de su investigación en el contexto cognitivo, expone los problemas o dificultades identificadas y plantea soluciones para avanzar en su exploración.

Segunda sesión.

1. Completar la segunda sección de comentarios del formulario de reflexión sobre la planificación y el progreso y enviarlo a su supervisor.

Tercera sesión.

2. Completar la última sección de comentarios en el formulario y enviarla a su supervisor.

3. El estudiante indica lo que logró y realiza preguntas para posibles investigaciones, qué aspectos literarios se podrían analizar y que se pudo trabajar de mejor manera o con un enfoque distinto.

4. El estudiante carga a la plataforma Turnitin su monografía y reflexiones para verificar probidad académica.

Fuente: Elaboración propia.

\section{b) Actividades del supervisor}

Primera y segunda sesión. - Poner sus iniciales y la fecha en el formulario. Revisar que se declare el trabajo realizado en la primera y segunda reflexión. Enviar retroalimentación de la primera y segunda reflexión, recomendaciones y sugerencias marcadas como comentario inserto en el documento enviado. Revisión de las correcciones sugeridas de cada reflexión. Comprobación del trabajo logrado e ideas planteadas con las directrices marcadas por la etapa de reflexión de acuerdo con la guía de monografía.

Tercera Sesión. - Revisión final del documento, identificando que se cumplan con todos los lineamientos marcados en el proceso con criterios de evaluación: Enfoque y método, conocimiento y comprensión, pensamiento crítico, presentación y compromiso. Confirmar la autoría original del formulario y de la monografía. Revisión de la versión final de monografía y reflexiones en la plataforma Turnitin. Enviar el formulario al Bachillerato Internacional junto con la monografía terminada. Subida del trabajo en la plataforma (IBIS) según fechas establecidas por la institución.

Adicionalmente, la Tabla 4 señala los tiempos que se utilizaron en la planificación inversa de acompañamiento docente para el avance de la construcción 
del trabajo de monografía de los estudiantes del Bachillerato Internacional (BI) en español categoría 1, a partir de la experiencia profesional.

Tabla 4:

Tiempos empleados en la propuesta de planificación inversa

Tiempo total recomendado por el (BI) para las 3 sesiones (minutos): $\quad$ Entre 180 y 300

Tiempo empleado por el supervisor de monografía (en minutos):

200

Sesión 1

60

Sesión 2

70

Sesión 3

70

Tiempos de aprendizaje invertido:

(a) Tiempo previsto para el aprendizaje autónomo (en días):

(b) Tiempo previsto para el co-aprendizaje (en minutos):

(c) Tiempo previsto para la sociabilización (en minutos):

90

Fuente: Elaboración propia.

\section{RESULTADOS}

La aplicación del modelo de aula invertida para la producción de la monografía como componente de evaluación externa en el programa del Bachillerato Internacional, permitió que los estudiantes que adoptaron esta oferta educativa en su formación consoliden habilidades de pensamiento y autogestión las mismas que pusieron en evidencia su capacidad para hacer deducciones y extraer conclusiones sobre aspectos lingüísticos y estilísticos de los textos literarios estudiados, transferir y aplicar los conocimientos existentes para generar nuevas ideas y atenerse a los horarios de clase y las fechas límites del proyecto.

Asimismo, desarrollaron competencias para convivir con el nuevo entorno de aprendizaje en el que se presenta su habilidad para encontrar y seleccionar información a través de diferentes medios, usando la tecnología como aliada para cumplir con este propósito. El proceso de monografía se desarrolló de forma adecuada y consolidó un proceso de enseñanza-aprendizaje en el que ubicó al estudiante como el protagonista en la adquisición y creación de nuevo conocimiento en el campo cognitivo y metacognitivo.

La aplicación de actividades sincrónicas y asincrónicas mediadas por la comunicación horizontal que presentó el aula invertida permitió que los dos alumnos alcancen una calificación de $\mathrm{B}$ y $\mathrm{C}$ en este componente de evaluación correspondiente a la convocatoria mayo 2020; por lo que su validez en el proceso también permitirá que su aplicación por las circunstancias sociales por las que atraviesa el país y el mundo también beneficie el proceso de monografía de los alumnos de la convocatoria mayo 2021 que cursan el programa del diploma en el Bachillerato Internacional.

La gestión del tiempo y planificación de actividades divididas en sesiones de reflexión y consulta demostraron que el alumno está apto para producir un trabajo de forma independiente con la guía y consejos de su supervisor, por esta razón se cumplen con las características del perfil de comunidad $\mathrm{Bl}$, y los estudiantes 
además de alcanzar habilidades y competencias se vinculan directamente en la práctica educativa y en la vida con atributos de indagadores, reflexivos, de pensamiento crítico y buenos comunicadores lo que refleja su audacia ante un desafío académico y social que modificó su forma de aprender y producir trabajos escritos.

\section{CONCLUSIONES}

El aula invertida permite establecer un ambiente dinámico, trabajo colaborativo, aprendizajes significativos y el desarrollo de habilidades de forma autónoma; como también favorece una actitud de compromiso y de interés por investigar conservando la motivación

El desarrollo de este trabajo muestra un resultado muy positivo, tanto en el proceso de aprendizaje como en la aceptación por parte de los alumnos, siendo claves el uso de las aulas invertidas para el proceso de aprendizaje, lo que evidencia la necesidad de la inclusión de este tipo de metodologías.

\section{REFERENCIAS}

Akçayır, G. y Akçayır, M. (2018). The flipped classroom: A review of its advantages and challenges. Computers and Education, 126(9), 334-345. Recuperado de: https://doi.org/10.1016/j.compedu.2018.07.021

Acosta, J.; Cid, J.; Silva, C. y Madariaga, E. (2020). Impacto y recomendaciones de clase invertida en el proceso de enseñanza-aprendizaje de geometría. Formación Universitaria, 13(3), 3-10. Recuperado de: https://doi.org/10.4067/s0718-50062020000300003

Castillo, M. y Montoya, J. (2015). Dinámica ideo-espiritual de la formación estéticopedagógica del docente. Alteridad, 10(2), pp. 190-204.

Castillo, M., Rivera, J. M., \& Seisdedos, L. F. (2013). Los sentimientos pedagógicos en el desempeño de los docentes del sistema educativo ecuatoriano. Didasc@ lia: Didáctica y Educación, 4(2), 101-116.

Collazos, C. (2006). How to take advantage of "cooperative learning" in the classroom. Educación y Educadores, Volumen 9(4128), 61-76.

Comunicado oficial | Medidas adoptadas con respecto a la oferta educativa de Bachillerato Internacional - Ministerio de Educación. (s/f). Recuperado de: https://educacion.gob.ec/comunicado-oficial-medidas-adoptadas-conrespecto-a-la-oferta-educativa-de-bachillerato-internacional/

Creamer, M. (2020, March 16). Plan Educativo Covid-19 - Ministerio de Educación. Recuperado de: https://educacion.gob.ec/plan-educativo-covid-19-sepresento-el-16-de-marzo/

Di Castri, F. (2010). El desarrollo en la sociedad de la información. In Anales de la Universidad de Chile 3(.1). Recuperado de: https://doi.org/10.5354/03657779.2004 .3318

Unday, D. y Valero, J. (2017). Sistematización de experiencias como método de investigación. Gaceta Médica Espirituana, 19(2).

Fernández, J.; Castro, E.; Estrella, M.; Fernández, E.; Rico, L.; Hidalgo, J. y Marín, M. (2016). Significados y concepciones de conceptos matemáticos escolares. Investigación En Educación Matemática XX, 237-246. Recuperado de: http://funes.uniandes.edu.co/8863/1/Castro2016Significado.pdf 
Granja, D. (2015). Constructivism as theory and teaching method. Sophia, 19(2), 93-110. recuperado de: https://doi.org/10.17163/soph.n19.2015.04

Guía para convertirse en bachiller: País: La Hora Noticias de Ecuador, sus provincias el mundo (n.d.). Recuperado de: https://lahora.com.ec/noticia/1101876487/noticia

Hernández, C., y Flores, S. (2017). Flipped classroom mediated by the use of virtual platforms: A case study of pre-service teacher education in physics | Aula invertida mediada por el uso de plataformas virtuales: Un estudio de caso en la formación de profesores de física. Estudios Pedagogicos, 43(3), 193-204.

Hernandez, R. (2017). Impacto de las TIC en la educación: Retos y Perspectivas. Propósitos y Representaciones, 5(1), 325. Recuperado de: https://doi.org/10.20511/pyr2017.v5n1.149

Hope, R., y Ca, (2016). Las herramientas de comunicación sincrónica y asincrónica en la clase presencial. 1-10.

Ibarra, A. y Llata, D. (2010). Niños nativos digitales en la sociedad del conocimiento: acercamientos conceptuales a sus competencias. Razón y Palabra, (72), 2624.

Maria, B. y Ovide, E. (2011). El impacto de las nuevas tecnologías en la educación en valores del siglo XXI1. (Spanish). Sinéctica, (37), 1-15. Recuperado de: http://search.ebscohost.com/login.aspx?direct=true\&db=a9h\&AN=70502704\&l ang $=$ es\&site $=$ ehost-live

Ministerio de Educación. (2020). Plan Educativo: aprendamos juntos en casa. Mineduc,53(91-19. Recuperado de: https://doi.org/10.101 7/CBO9781107415324.004

Ministra de Educación explica el plan educativo virtual por el Covid-19. (2020). Recuperado de: https://gk.city/2020/03/16/plan-educativo-virtual-covid19/

Organización del Bachillerato Internacional. (2017). Guía Monografía. 385.

Rojas, N.; Pérez, F.; Torres, I. y Peláez, E. (2014). Las aulas virtuales: una opción para el desarrollo de la Educación Médica. Educación Médica Del Centro, $6(2), 231-247$.

Santoveña, I. (2017). El proceso de enseñanza-aprendizaje a través de herramientas de comunicación síncrona: El caso de Elluminate Live. Electronic Journal of Research in Education Psychology, 10(26). Recuperado de: https://doi.org/10.25115/ejrep.v10i26.1508

Ser Bachiller: Desde su inicio la prueba ha tenido cinco cambios. (n.d.). Recuperado de: https://www.expreso.ec/guayaquil/bachiller-inicio-prueba-tenido-cincocambios-3392.html

Yépez, A. y Castillo, M. (2020). La inclusión educativa en la Universidad Central del Ecuador desde las percepciones del estudiantado. Retos de la Ciencia. 4(1), pp. 114 